\title{
Sphericalization and $p$-harmonic functions on unbounded domains in Ahlfors regular metric spaces
}

\author{
Anders Björn \\ Department of Mathematics, Linköping University, \\ SE-58183 Linköping, Sweden; anders.bjorn@liu.se \\ Jana Björn \\ Department of Mathematics, Linköping University, \\ SE-58183 Linköping, Sweden; jana.bjorn@liu.se \\ Xining Li \\ Department of Mathematics, Sun Yat-sen University, \\ Guangzhou, 510275, P.R. China; E-mail address: lixining3@mail.sysu.edu.cn
}

\begin{abstract}
We use sphericalization to study the Dirichlet problem, Perron solutions and boundary regularity for $p$-harmonic functions on unbounded sets in Ahlfors regular metric spaces. Boundary regularity for the point at infinity is given special attention. In particular, we allow for several "approach directions" towards infinity and take into account the massiveness of their complements.

In 2005, Llorente-Manfredi-Wu showed that the $p$-harmonic measure on the upper half space $\mathbf{R}_{+}^{n}, n \geq 2$, is not subadditive on null sets when $p \neq 2$. Using their result and spherical inversion, we create similar bounded examples in the unit ball $\mathbf{B} \subset \mathbf{R}^{n}$ showing that the $n$-harmonic measure is not subadditive on null sets when $n \geq 3$, and neither are the $p$-harmonic measures in $\mathbf{B}$ generated by certain weights depending on $p \neq 2$ and $n \geq 2$.
\end{abstract}

Key words and phrases: Ahlfors regular metric space, boundary regularity, Dirichlet problem, Muckenhoupt $A_{p}$ weight, Perron solution, $p$-harmonic function, $p$-harmonic measure, Poincaré inequality, sphericalization.

Mathematics Subject Classification (2010): Primary: 31E05; Secondary: 26D10, 30L99, 35J66, 35J92, 49Q20.

\section{Introduction}

In this paper we use sphericalization to study Perron solutions and boundary regularity for $p$-harmonic functions on unbounded sets in metric measure spaces. On unweighted $\mathbf{R}^{n}$, a $p$-harmonic function in an open set $\Omega \subset \mathbf{R}^{n}$ is a continuous weak solution $u$ of the $p$-Laplace equation

$$
\Delta_{p} u=\operatorname{div}\left(|\nabla u|^{p-2} \nabla u\right)=0 .
$$

Equivalently, $u$ is a continuous minimizer of the $p$-energy integral among functions with the same boundary values, i.e.

$$
\int_{\varphi \neq 0}|\nabla u|^{p} d x \leq \int_{\varphi \neq 0}|\nabla(u+\varphi)|^{p} d x \quad \text { for all } \varphi \in \operatorname{Lip}_{c}(\Omega) .
$$


This latter definition is suitable for generalization to metric spaces, using $p$-weak upper gradients.

The setting considered in this paper is a complete metric space $(X, d)$ equipped with an Ahlfors $Q$-regular measure $\mu, Q>1$, supporting a $q$-Poincaré inequality. The precise relations between $p, Q$ and $q$ are given in (5.1) and (5.2) and make it possible to "sphericalize" $X$ into a new bounded metric space with suitable properties, where the theory of $p$-harmonic functions is well-developed.

Our primary object of study is the Dirichlet problem, which given $f: \partial \Omega \rightarrow \mathbf{R}$ asks for a $p$-harmonic function having $f$ as boundary values in some weak sense. We use the Perron method to solve this problem, which always provides two solutions $\underline{P} f \leq \bar{P} f$ of the Dirichlet problem. When they coincide we denote the common solution by $P f$ and $f$ is called resolutive. Under the assumptions in this paper, continuous functions are resolutive. A boundary point $x_{0} \in \partial \Omega$ is regular if

$$
\lim _{\Omega \ni y \rightarrow x} P f(y)=f(x) \text { for all } f \in C(\partial \Omega) .
$$

Perron solutions for $p$-harmonic functions on bounded open sets $\Omega$ in metric spaces were first studied in Björn-Björn-Shanmugalingam [12]. Boundary regularity for $p$-harmonic functions on bounded open sets in metric spaces was studied even earlier in Björn [18], Björn-MacManus-Shanmugalingam [22] and BjörnBjörn-Shanmugalingam [11]; and a rather extensive study was carried out in BjörnBjörn [7].

In this paper, our main interest lies in proving resolutivity and boundary regularity in unbounded $\Omega$ by using sphericalization. The sphericalization technique makes it possible to map the unbounded space $X$ into a bounded metric space, while preserving the $p$-harmonic functions, in the spirit of the Kelvin transform. We can then appeal to the earlier results for bounded sets and "map" them back to the original unbounded situation. When $\Omega$ is unbounded we consider its boundary within the one-point compactification of $X$, so $\infty$ is included in the boundary.

Perron solutions for $p$-harmonic functions on unbounded open sets in metric spaces have been studied by Hansevi [28] who realised that there were substantial additional complications in the unbounded case compared with the bounded case. There is some overlap between his and our results. To our best knowledge, boundary regularity for $p$-harmonic functions on unbounded open sets in metric spaces has not been studied before, beyond weighted $\mathbf{R}^{n}$. However, regularity of the boundary at $\infty$ for global $p$-harmonic functions on certain Cartan-Hadamard manifolds and Gromov hyperbolic spaces was considered in e.g. Holopainen [32], Holopainen-Lang-Vähäkangas [33] and Holopainen-Vähäkangas [34] .

Li-Shanmugalingam [44] showed that (under suitable conditions) sphericalization preserves Ahlfors regularity and Poincaré inequalities. These significant results are our starting point. The measure $\mu_{a}$, with which they equipped the sphericalization, does however not preserve the $p$-energy, and we are therefore forced to equip the sphericalization with a different measure $\hat{\mu}$, generated by a suitable power weight. Using (a metric space version of) the theory of Muckenhoupt $A_{p}$ weights we can show that the sphericalization equipped with $\hat{\mu}$ supports a $p$-Poincaré inequality, provided the original space supports a $q$-Poincaré inequality, with $q$ given by (5.2). This whole machinery is carried out in Sections $2-5$.

In Section 6 we are then ready to start our study of $p$-harmonic functions and especially Perron solutions on unbounded sets. We obtain several resolutivity and perturbation results, see Theorems 6.5 and 6.7. We also show that boundary regularity is a local property, that it can be characterized using barriers, and that the Kellogg property holds, i.e. the set of irregular boundary points has capacity zero.

Since regularity is a local property, many of the results on boundary regularity for bounded sets, such as the Wiener criterion, carry over directly to finite boundary 
points of unbounded sets. The point at $\infty$, however, requires special attention and is studied in Section 7.

In unweighted $\mathbf{R}^{n}, n \geq 2$, with $p>n / 2$ (in particular in $\mathbf{R}^{2}$ for any $p>1$ ) we get a number of new results. The resolutivity of continuous functions, the Kellogg property and the barrier characterization have been known before in this setting, see Kilpeläinen [37, Theorems 1.5, 1.10 and Corollary 5.6]. The obtained resolutivity results for Newtonian (and Dirichlet) functions are new when $n / 2<p<n$, while for $p \geq n$ they were proved in Hansevi [28] using different methods. Theorem 6.6 is new for all $p>n / 2$, although [28] contains a weaker result for unbounded $p$ parabolic sets. We also obtain several new characterizations of boundary regularity in unbounded sets, corresponding to the results in Björn-Björn [7, Theorem 6.1]; see also Heinonen-Kilpeläinen-Martio [29, Chapter 9].

Since the $p$-energy is preserved, also quasiminimizers are preserved in just the same way as $p$-harmonic functions. Hence, many earlier boundary regularity results for quasiminimizers generalize from bounded to unbounded sets, see the end of Section 6. It also follows that sphericalization is a quasiconformal mapping, by Theorem 4.1 in Korte-Marola-Shanmugalingam [41].

In unbounded domains, the point at infinity can often be approached from different directions, e.g. in an infinite strip or cylinder. Also on bounded domains, rather than using the given metric boundary, there are many situations where one would like to distinguish between different directions towards a boundary point. For example, in the slit disc, where a horizontal ray is removed from the disc, it is natural to consider different boundary values along the ray from above and from below. Björn-Björn-Shanmugalingam [13] carried out such a study using the Mazurkiewicz metric on bounded domains in metric spaces. We are now able to transfer these results to unbounded domains, and we explain in particular what happens at infinity in Section 7. We also provide an example where the point at $\infty$ corresponds to uncountably many directions, i.e. boundary points with respect to the Mazurkiewicz metric, and yet behaves well for the Perron method, see Example 7.5.

Llorente-Manfredi-Wu [45], using a very sophisticated argument due to Wolff [50], showed that for any $p \neq 2$ and $n \geq 2$, there are sets $A_{1}, A_{2} \subset \mathbf{R}^{n-1}$ such that

$$
\omega_{p}\left(A_{1} ; \mathbf{R}_{+}^{n}\right)=\omega_{p}\left(A_{2} ; \mathbf{R}_{+}^{n}\right)=0<\omega_{p}\left(A_{1} \cup A_{2} ; \mathbf{R}_{+}^{n}\right),
$$

where $\omega_{p}\left(\cdot ; \mathbf{R}_{+}^{n}\right)$ denotes the $p$-harmonic measure with respect to the upper half space $\mathbf{R}_{+}^{n}$. Despite its name, $\omega_{p}\left(\cdot ; \mathbf{R}_{+}^{n}\right)$ is evidently not a measure, but rather a nonlinear generalization of the harmonic measure, and (1.1) shows that it is not even subadditive. In Section 8 we transfer the examples from [45] to the unit ball in $\mathbf{R}^{n}$ and thus create similar bounded examples with respect to a weighted measure depending on $p$ and $n$. In particular, the measure is the usual Lebesgue measure (without weight) when $p=n$, so we obtain an analogue of (1.1) for the usual $n$-harmonic measure for the $n$-Laplacian in the unit ball.

Acknowledgement. The first two authors were supported by the Swedish Research Council grants 2016-03424 and 621-2014-3974, respectively. The third author was supported by NSFC grant 11701582 . Part of the research was done during two visits of the third author to Linköping University in 2016 and 2018.

\section{Metric spaces and power weights}

We assume throughout the paper that $1<p<\infty$ and that $X=(X, d, \mu)$ is a metric space equipped with a metric $d$ and a positive complete Borel measure $\mu$ such that $0<\mu(B)<\infty$ for all balls $B \subset X$. Additional standing assumptions will be given at the beginning of Sections 3 and 5 . Proofs of the results in this 
section can be found in the monographs Björn-Björn [9] and Heinonen-KoskelaShanmugalingam-Tyson [31].

The measure $\mu$ is doubling if there exists $C>0$ such that for all balls $B=$ $B\left(x_{0}, r\right):=\left\{x \in X: d\left(x, x_{0}\right)<r\right\}$ in $X$,

$$
0<\mu(2 B) \leq C \mu(B)<\infty
$$

Here and elsewhere $\lambda B=B\left(x_{0}, \lambda r\right)$. A metric space with a doubling measure is proper (i.e. closed bounded subsets are compact) if and only if it is complete.

A curve is a continuous mapping from an interval, and a rectifiable curve is a curve with finite length. Unless otherwise mentioned, we will only consider curves which are nonconstant, compact and rectifiable, and thus each curve can be parameterized by its arc length $d s$. For a family $\Gamma$ of curves in $X$, we define its $p$-modulus

$$
\operatorname{Mod}_{p}(\Gamma):=\inf \int_{X} \rho^{p} d \mu
$$

where the infimum is taken over all Borel functions $\rho \geq 0$ such that $\int_{\gamma} \rho d s \geq 1$ for all $\gamma \in \Gamma$. A property is said to hold for $p$-almost every curve if it fails only for a curve family $\Gamma$ with zero $p$-modulus. Following Heinonen-Koskela [30], we introduce upper gradients as follows (they called them very weak gradients).

Definition 2.1. A Borel function $g: X \rightarrow[0, \infty]$ is an upper gradient of a function $u: X \rightarrow \overline{\mathbf{R}}:=[-\infty, \infty]$ if for all curves $\gamma:\left[0, l_{\gamma}\right] \rightarrow X$,

$$
\left|u(\gamma(0))-u\left(\gamma\left(l_{\gamma}\right)\right)\right| \leq \int_{\gamma} g d s
$$

where the left-hand side is interpreted as $\infty$ whenever at least one of the terms therein is infinite. If $g: X \rightarrow[0, \infty]$ is measurable and $(2.1)$ holds for $p$-almost every curve, then $g$ is a $p$-weak upper gradient of $u$.

The $p$-weak upper gradients were introduced in Koskela-MacManus [42]. It was also shown therein that if $g \in L_{\text {loc }}^{p}(X)$ is a $p$-weak upper gradient of $u$, then one can find a sequence $\left\{g_{j}\right\}_{j=1}^{\infty}$ of upper gradients of $u$ such that $\left\|g_{j}-g\right\|_{L^{p}(X)} \rightarrow 0$. If $u$ has an upper gradient in $L_{\text {loc }}^{p}(X)$, then it has an a.e. unique minimal $p$-weak upper gradient $g_{u} \in L_{\mathrm{loc}}^{p}(X)$ in the sense that $g_{u} \leq g$ a.e. for every $p$-weak upper gradient $g \in L_{\mathrm{loc}}^{p}(X)$ of $u$, see Shanmugalingam [48].

Together with the doubling property defined above, the following Poincaré inequality is often a standard assumption on metric spaces.

Definition 2.2. We say that $X$ (or $\mu$ ) supports a $q$-Poincaré inequality, $q \geq 1$, if there exist constants $C>0$ and $\lambda \geq 1$ such that for all balls $B \subset X$, all integrable functions $u$ on $X$ and all ( $q$-weak) upper gradients $g$ of $u$,

$$
f_{B}\left|u-u_{B}\right| d \mu \leq C \operatorname{diam}(B)\left(f_{\lambda B} g^{q} d \mu\right)^{1 / q}
$$

where $u_{B}:=f_{B} u d \mu:=\int_{B} u d \mu / \mu(B)$.

As is customary, we say that $A \lesssim B$ (and equivalently $B \gtrsim A$ ), if there is a constant $C>0$ (independent of the variables that $A$ and $B$ are functions of) such that $A \leq C B$. We also write $A \simeq B$ if $A \lesssim B \lesssim A$.

With this notation, $\mu$ is Ahlfors $Q$-regular if

$$
\mu(B(x, r)) \simeq r^{Q} .
$$


Ahlfors regularity is a relatively strong assumption on the measure. At the same time, it is easily verified that doubling measures in connected spaces satisfy the one-sided estimates

$$
\left(\frac{r^{\prime}}{r}\right)^{s} \lesssim \frac{\mu\left(B\left(x^{\prime}, r^{\prime}\right)\right)}{\mu(B(x, r))} \lesssim\left(\frac{r^{\prime}}{r}\right)^{\sigma}
$$

for some $0<\sigma \leq s<\infty$, all $x \in X, x^{\prime} \in B(x, r)$ and all $0<r^{\prime} \leq r \leq 2 \operatorname{diam} X$.

The following proposition makes it possible to construct new well-behaved measures from old ones.

Proposition 2.3. Let $X$ be a metric space equipped with a doubling measure $\mu$ such that for some $\sigma>0, c \in X$ and all $0<r^{\prime} \leq r \leq 2 \operatorname{diam} X$,

$$
\frac{\mu\left(B\left(c, r^{\prime}\right)\right)}{\mu(B(c, r))} \lesssim\left(\frac{r^{\prime}}{r}\right)^{\sigma}
$$

Let $\alpha \in \mathbf{R}$ and $w(x)=d(x, c)^{\alpha}$. Then the following are true for all balls $B \subset X$, with comparison constants depending on the one in (2.2), as well as on $\alpha$ and $\sigma$ :

(a) If $p>1$ and $-\sigma<\alpha<\sigma(p-1)$, then

$$
f_{B} w d \mu\left(f_{B} w^{1 /(1-p)} d \mu\right)^{p-1} \lesssim 1
$$

(b) If $-\sigma<\alpha \leq 0$, then

$$
f_{B} w d \mu \lesssim \underset{B}{\operatorname{essinf}} w
$$

If (2.3) holds, we say that $w$ is an $A_{p}$ weight with respect to $\mu$, while if (2.4) holds then $w$ is an $A_{1}$ weight. We also write $w \in A_{p}(\mu), p \geq 1$, in these cases.

Proof. We shall distinguish between three types of balls:

1. Let $B=B(c, r), 0<r \leq 2 \operatorname{diam} X$. Then for all $\alpha>-\sigma$,

$$
\begin{aligned}
\int_{B} d(x, c)^{\alpha} d \mu & \simeq \sum_{j=0}^{\infty}\left(2^{-j} r\right)^{\alpha} \mu\left(2^{-j} B \backslash 2^{-j-1} B\right) \\
& \lesssim \sum_{j=0}^{\infty}\left(2^{-j} r\right)^{\alpha}\left(2^{-j}\right)^{\sigma} \mu(B) \simeq r^{\alpha} \mu(B)
\end{aligned}
$$

Replacing $\alpha$ by $\alpha /(1-p)>-\sigma$ in $(2.5)$ we obtain

$$
\left(f_{B} w^{1 /(1-p)} d \mu\right)^{p-1} \lesssim\left(r^{\alpha /(1-p)}\right)^{p-1}=r^{-\alpha} .
$$

Together with (2.5), this yields $(2.3)$ for $B=B(c, r)$. To prove $(2.4)$ for such $B$, it suffices to note that for all $\alpha \leq 0$,

$$
\underset{B}{\operatorname{essinf}} d(x, c)^{\alpha} \geq r^{\alpha} .
$$

2. If $B=B(z, r)$ and $r>\frac{1}{2} d(z, c)$ then $B \subset B(c, 3 r)$ and $\mu(B) \simeq \mu(B(c, 3 r))$, by the doubling property of $\mu$. We can therefore replace $B$ by $B(c, 3 r)$ in $(2.3)$ and (2.4) as follows, using case 1,

$$
f_{B} w d \mu\left(f_{B} w^{1 /(1-p)} d \mu\right)^{p-1} \lesssim f_{B(c, 3 r)} w d \mu\left(f_{B(c, 3 r)} w^{1 /(1-p)} d \mu\right)^{p-1} \lesssim 1
$$


and

$$
f_{B} w d \mu \lesssim f_{B(c, 3 r)} w d \mu \lesssim \underset{B(c, 3 r)}{\operatorname{essinf}} w \leq \underset{B}{\operatorname{essinf}} w .
$$

3. If $B=B(z, r)$ and $0<r \leq \frac{1}{2} d(z, c)$ then $w(x)=d(x, c)^{\alpha} \simeq d(z, c)^{\alpha}$ for all $x \in B$ and hence

$$
f_{B} w d \mu \simeq d(z, c)^{\alpha} \quad \text { and } \quad f_{B} w^{1 /(1-p)} d \mu \simeq d(z, c)^{\alpha /(1-p)},
$$

from which (2.3) follows. Similarly,

$$
f_{B} w d \mu \simeq d(z, c)^{\alpha} \simeq \underset{B}{\operatorname{essinf}} w
$$

Proposition 2.3 can be combined with Theorem 4 in Björn [17] (whose proof works also in metric spaces) to obtain the following result.

Corollary 2.4. Assume that $\mu$ is doubling, satisfies (2.2) and supports a q-Poincaré inequality on $X$. Then the measure $d \nu=d(x, c)^{\alpha} d \mu$, with $-\sigma<\alpha \leq 0$, is also doubling and supports a q-Poincaré inequality. For $\alpha>0$ it is doubling and supports a $q^{\prime}$-Poincaré inequality for every $q^{\prime}>q(1+\alpha / \sigma)$.

\section{Sphericalization}

From now on we assume that $(X, d, \mu)$ is complete and unbounded.

Following Li-Shanmugalingam [44] we will now define the sphericalization of $X$. Let $\widehat{X}=X \cup\{\infty\}$ be the one-point compactification of $X$. We also fix a base point $a \in X$ from now on. Define $d_{a}, \hat{d}: \widehat{X} \times \widehat{X} \rightarrow[0, \infty)$ by

$$
d_{a}(x, y)=d_{a}(y, x)= \begin{cases}\frac{d(x, y)}{(1+d(x, a))(1+d(y, a))}, & \text { if } x, y \in X \\ \frac{1}{1+d(x, a)}, & \text { if } x \in X \text { and } y=\infty, \\ 0, & \text { if } x=y=\infty\end{cases}
$$

and

$$
\hat{d}(x, y)=\inf _{\left(x=x_{0}, x_{1}, \ldots, x_{k}=y\right)} \sum_{j=1}^{k} d_{a}\left(x_{j}, x_{j-1}\right),
$$

where the infimum is over all finite sequences $\left(x=x_{0}, x_{1}, \ldots, x_{k}=y\right)$. This makes $\hat{d}$ into a metric on $\widehat{X}$, and $(\widehat{X}, \hat{d})$ is the sphericalization of $(X, d)$. Moreover,

$$
\frac{1}{4} d_{a}(x, y) \leq \hat{d}(x, y) \leq d_{a}(x, y)
$$

see (3.2) in Buckley-Herron-Xie [24] and the proof of Lemma 2.2 in Bonk-Kleiner [23]. Note that $d_{a}$ is in general not a metric since the triangle inequality may fail for it. We will denote balls in $(\widehat{X}, \hat{d})$ by $\widehat{B}(x, r)$.

In Li-Shanmugalingam [44], the sphericalization $(\widehat{X}, \hat{d})$ is equipped with the measure $\mu_{a}$ defined by

$$
\mu_{a}(A)=\int_{A \backslash\{\infty\}} \frac{d \mu(x)}{\mu(B(a, 1+d(x, a)))^{2}} .
$$

Proposition 3.1. It is always true that $\mu_{a}(\widehat{X})<\infty$. 
Proof. Let $b_{j}=\mu(B(a, j))$ (with $\left.b_{0}=0\right)$. Then

$$
\mu_{a}(\widehat{X}) \leq \sum_{j=0}^{\infty} \frac{b_{j+1}-b_{j}}{b_{j+1}^{2}} \leq \frac{1}{b_{1}}+\sum_{j=1}^{\infty} \frac{b_{j+1}-b_{j}}{b_{j} b_{j+1}}=\frac{1}{b_{1}}+\sum_{j=1}^{\infty}\left(\frac{1}{b_{j}}-\frac{1}{b_{j+1}}\right) \leq \frac{2}{b_{1}}
$$
by

In this paper it will be more useful to equip $(\widehat{X}, \hat{d})$ with the measure $\hat{\mu}$ defined

$$
\hat{\mu}(A)=\int_{A \backslash\{\infty\}} \frac{d \mu(x)}{(1+d(x, a))^{2 p}} .
$$

In order to use the results from [44] we will need to carefully study the connections between the measures $\mu_{a}$ and $\hat{\mu}$, which we do in Section 5. For the rest of this section we will only use the measure $\hat{\mu}$ on $\widehat{X}$. Note that $\hat{\mu}$ depends on $p$, even though this is not made explicit in the notation.

The measure $\hat{\mu}(\widehat{X})$ can be either finite or infinite. Strictly speaking, as $\widehat{X}$ is bounded, to fall within the scope of the theory considered e.g. in [9] we would need to require that $\hat{\mu}(\widehat{X})<\infty$, but the results in the rest of this section, as well as in Section 4 , remain valid also in the case when $\hat{\mu}(\widehat{X})=\infty$. Under the assumptions at the beginning of Section 5 it follows from Proposition 5.1 that $\hat{\mu}(\widehat{X})<\infty$.

If $\gamma:[0,1] \rightarrow X$ is a (not necessarily rectifiable) curve, then we can consider its length with respect to $d$ and with respect to $\hat{d}$. It is quite easy (cf. Li-Shanmugalingam [44]) to see that the arc lengths $d s$ and $d \hat{s}_{a}$ with respect to $d$ and $\hat{d}$, respectively, are related by

$$
d \hat{s}_{a}(x)=\frac{d s(x)}{(1+d(x, a))^{2}} .
$$

As $\gamma([0,1])$ is compact it follows that $\gamma$ is rectifiable with respect to $d$ if and only if it is rectifiable with respect to $\hat{d}$.

Lemma 3.2. Let $\Gamma$ be a collection of rectifiable curves on $X$. Then

$$
\operatorname{Mod}_{p}(\Gamma ; X, d, \mu)=\operatorname{Mod}_{p}(\Gamma ; \widehat{X}, \hat{d}, \hat{\mu}) .
$$

Proof. Let $\rho$ be a nonnegative Borel function which is admissible in the definition of $\operatorname{Mod}_{p}(\Gamma ; X, d, \mu)$, i.e. $\int_{\gamma} \rho d s \geq 1$ for all $\gamma \in \Gamma$. Let $\hat{\rho}(x)=\rho(x)(1+d(x, a))^{2}$. Then, by (3.3),

$$
\int_{\gamma} \hat{\rho} d \hat{s}_{a}=\int_{\gamma} \rho d s \geq 1
$$

and thus $\hat{\rho}$ is admissible in the definition of $\operatorname{Mod}_{p}(\Gamma ; \widehat{X}, \hat{d}, \hat{\mu})$. Moreover,

$$
\int_{\widehat{X}} \hat{\rho}^{p} d \hat{\mu}=\int_{X} \rho(x)^{p}(1+d(x, a))^{2 p} \frac{d \mu(x)}{(1+d(x, a))^{2 p}}=\int_{X} \rho^{p} d \mu .
$$

Taking infimum over all such $\rho$ shows that $\operatorname{Mod}_{p}(\Gamma ; \widehat{X}, \hat{d}, \hat{\mu}) \leq \operatorname{Mod}_{p}(\Gamma ; X, d, \mu)$. The converse inequality is shown similarly.

Lemma 3.3. Let $\Omega \subset X$ be open, $u: \Omega \rightarrow \overline{\mathbf{R}}$ be a function, $g: \Omega \rightarrow[0, \infty]$ be measurable, and

$$
\hat{g}(x)=g(x)(1+d(x, a))^{2}, \quad x \in \Omega .
$$

Then $g$ is a p-weak upper gradient of $u$ in $\Omega$ with respect to $(d, \mu)$ if and only if $\hat{g}$ is a p-weak upper gradient of $u$ in $\Omega$ with respect to $(\hat{d}, \hat{\mu})$.

Observe that measurability is the same with respect to $\mu$ and $\hat{\mu}$. 
Proof. Assume that $g$ is a $p$-weak upper gradient of $u$ with respect to $(d, \mu)$. Let $\Gamma$ be the family of exceptional curves in $\Omega$ for which (2.1) fails. Then $\operatorname{Mod}_{p}(\Gamma ; X, d, \mu)=$ 0 . Let $\gamma:\left[0, l_{\gamma}\right] \rightarrow \Omega$ be a curve not in $\Gamma$. Then, using (3.3),

$$
\left|u(\gamma(0))-u\left(\gamma\left(l_{\gamma}\right)\right)\right| \leq \int_{\gamma} g d s=\int_{\gamma} \hat{g} d \hat{s}_{a} .
$$

As $\operatorname{Mod}_{p}(\Gamma ; \widehat{X}, \hat{d}, \hat{\mu})=\operatorname{Mod}_{p}(\Gamma ; X, d, \mu)=0$, by Lemma 3.2, we have shown that $\hat{g}$ is a $p$-weak upper gradient of $u$ with respect to $(\hat{d}, \hat{\mu})$. The converse implication is shown similarly.

\section{Newtonian spaces and capacity}

Following Shanmugalingam [47], we define a version of Sobolev spaces on the metric space $X$.

Definition 4.1. For a measurable function $u: X \rightarrow \overline{\mathbf{R}}$, let

$$
\|u\|_{N^{1, p}(X)}=\left(\int_{X}|u|^{p} d \mu+\inf _{g} \int_{X} g^{p} d \mu\right)^{1 / p}
$$

where the infimum is taken over all upper gradients $g$ of $u$. The Newtonian space on $X$ is

$$
N^{1, p}(X)=\left\{u:\|u\|_{N^{1, p}(X)}<\infty\right\} .
$$

The space $N^{1, p}(X) / \sim$, where $u \sim v$ if and only if $\|u-v\|_{N^{1, p}(X)}=0$, is a Banach space and a lattice, see Shanmugalingam [47]. We also define

$$
D^{p}(X)=\left\{u: u \text { is measurable and has an upper gradient in } L^{p}(X)\right\} .
$$

In this paper we assume that functions in $N^{1, p}(X)$ and $D^{p}(X)$ are defined everywhere (with values in $\overline{\mathbf{R}}$ ), not just up to an equivalence class in the corresponding function space. This is important for upper gradients to make sense.

For a measurable set $E \subset X$, the Newtonian space $N^{1, p}(E)$ is defined by considering $\left(E,\left.d\right|_{E},\left.\mu\right|_{E}\right)$ as a metric space in its own right. We say that $u \in N_{\text {loc }}^{1, p}(E)$ if for every $x \in E$ there exists a ball $B_{x} \ni x$ such that $u \in N^{1, p}\left(B_{x} \cap E\right)$. The spaces $D^{p}(E)$ and $D_{\text {loc }}^{p}(E)$ are defined similarly.

Definition 4.2. The (Sobolev) capacity of an arbitrary set $E \subset X$ is

$$
C_{p}(E)=\inf _{u}\|u\|_{N^{1, p}(X)}^{p}
$$

where the infimum is taken over all $u \in N^{1, p}(X)$ such that $u \geq 1$ on $E$.

We say that a property holds quasieverywhere (q.e.) if the set of points for which the property fails has capacity zero. The capacity is the correct gauge for distinguishing between two Newtonian functions. If $u \in N^{1, p}(X)$, then $u \sim v$ if and only if $u=v$ q.e. Moreover, if $u, v \in D_{\mathrm{loc}}^{p}(X)$ and $u=v$ a.e., then $u=v$ q.e.

Proposition 4.3. Let $\Omega \subset X$ be open and $u: \Omega \rightarrow \overline{\mathbf{R}}$ be measurable. Then $u \in D_{\mathrm{loc}}^{p}(\Omega, d, \mu)$ if and only if $u \in D_{\mathrm{loc}}^{p}(\Omega, \hat{d}, \hat{\mu})$, and in this case

$$
\hat{g}_{u}(x)=g_{u}(x)(1+d(x, a))^{2} \text { for a.e. } x \in \Omega,
$$

where $g_{u}$ and $\hat{g}_{u}$ are the minimal $p$-weak upper gradients with respect to $(d, \mu)$ and $(\hat{d}, \hat{\mu})$, respectively. Moreover,

$$
\int_{\Omega} g_{u}^{p} d \mu=\int_{\Omega} \hat{g}_{u}^{p} d \hat{\mu},
$$

and thus $D^{p}(\Omega, d, \mu)=D^{p}(\Omega, \hat{d}, \hat{\mu})$. 
Proof. If $u \in D^{p}(\Omega, d, \mu)$ has a minimal $p$-weak upper gradient $g_{u}$, then $\hat{g}:=$ $g_{u}(x)(1+d(x, a))^{2}$ is a $p$-weak upper gradient of $u$ with respect to $(\hat{d}, \hat{\mu})$, by Lemma 3.3. Moreover,

$$
\int_{\Omega} \hat{g}^{p} d \hat{\mu}=\int_{\Omega} g_{u}(x)^{p}(1+d(x, a))^{2 p} \frac{d \mu(x)}{(1+d(x, a))^{2 p}}=\int_{\Omega} g_{u}^{p} d \mu .
$$

Thus $u \in D^{p}(\Omega, \hat{d}, \hat{\mu})$ and $\hat{g}_{u} \leq \hat{g}$ a.e. The converse inequality is shown similarly, and hence $\hat{g}_{u}=\hat{g}$ a.e.

The local case follows since the minimal $p$-weak upper gradient only depends on the function locally, see [9, Lemma 2.23 and Remark 2.28].

For $N^{1, p}$ we have the following corresponding result.

Proposition 4.4. Let $\Omega \subset X$ be open and $u: \Omega \rightarrow \overline{\mathbf{R}}$ be measurable. Then the following are true:

(a) $\|u\|_{N^{1, p}(\Omega, \hat{d}, \hat{\mu})} \leq\|u\|_{N^{1, p}(\Omega, d, \mu)}$ and thus $N^{1, p}(\Omega, d, \mu) \subset N^{1, p}(\Omega, \hat{d}, \hat{\mu})$.

(b) If $\Omega$ is bounded, then $N^{1, p}(\Omega, d, \mu)=N^{1, p}(\Omega, \hat{d}, \hat{\mu})$, as sets but with comparable norms (depending on $\Omega$ ).

(c) $N_{\mathrm{loc}}^{1, p}(\Omega, d, \mu)=N_{\mathrm{loc}}^{1, p}(\Omega, \hat{d}, \hat{\mu})$.

Proof. Clearly, (c) follows directly from (b). To prove (b), we note that because of Proposition 4.3, $u \in D^{p}(\Omega, d, \mu)$ if and only if $u \in D^{p}(\Omega, \hat{d}, \hat{\mu})$, with equal seminorms (4.1). Since $\Omega$ is bounded, we also have that $1+d(x, a) \simeq 1$ for all $x \in \Omega$, which implies that

$$
\int_{\Omega}|u|^{p} d \mu \simeq \int_{\Omega}|u|^{p} d \hat{\mu}
$$

with comparison constants depending on $\Omega$. Thus $u \in N^{1, p}(\Omega, d, \mu)$ if and only if $u \in N^{1, p}(\Omega, \hat{d}, \hat{\mu})$ with comparable norms.

Finally, (a) follows immediately from (4.1) and the fact that $d \hat{\mu} \leq d \mu$.

We shall write $\widehat{C}_{p}$ and $C_{p}$ for the capacities associated with the spaces $(\widehat{X}, \hat{d}, \hat{\mu})$ and $(X, d, \mu)$, respectively.

Lemma 4.5. Let $E \subset X$. Then $\widehat{C}_{p}(E)=0$ if and only if $C_{p}(E)=0$.

Proof. The inequality $\widehat{C}_{p}(E) \lesssim C_{p}(E)$ follows directly from Proposition 4.4 (a).

Conversely, assume that $\widehat{C}_{p}(E)=0$. By Proposition $4.4(\mathrm{~b})$, the $N^{1, p}$-norms are comparable in $B(a, 2 j), j \geq 1$, from which it follows that $C_{p}(E \cap B(a, j))=0$, see e.g. Lemma 2.24 in [9]. The countable subadditivity of the capacity then shows that $C_{p}(E)=0$.

\section{Poincaré inequalities under sphericalization}

From now on we assume that $(X, d, \mu)$ is complete and unbounded, and that $\mu$ is Ahlfors $Q$-regular with $Q>1$. We also assume that

$$
\begin{cases}1<p<Q /(2-Q), & \text { if } 1<Q<2, \\ p>Q / 2, & \text { if } Q \geq 2\end{cases}
$$

and that $(X, d, \mu)$ supports a q-Poincaré inequality, where

$$
q= \begin{cases}p, & \text { if } Q / 2<p \leq Q, \\ p Q /(2 p-Q), & \text { if } p \geq Q\end{cases}
$$


These assumptions are satisfied e.g. if $X=\mathbf{R}^{n}, n \geq 2$, equipped with the Lebesgue measure, and $p>n / 2$. In particular, all $p>1$ are allowed in $\mathbf{R}^{2}$.

For every $Q>1$, Laakso [43] constructed a complete bounded Ahlfors $Q$-regular metric space supporting a 1-Poincaré inequality. Since it is bounded it does not fall within our scope here, but its flattening is an unbounded complete Ahlfors $Q$ regular metric space supporting a 1-Poincaré inequality, see Proposition 4.1 and Theorem 4.4 in Li-Shanmugalingam [44] and Theorem 3.3 in Korte [40].

Since $X$ is complete and $\mu$ is doubling and supports a Poincaré inequality, it follows that $(X, d)$ is quasiconvex, i.e. there is a constant $C \geq 1$ such that each pair of points $x$ and $y$ in the space can be joined by a curve $\gamma$ with length

$$
l_{\gamma} \leq C d(x, y)
$$

see e.g. Theorem 4.32 in [9]. Moreover, $1<q \leq Q$ and thus Theorem 3.3 in Korte [40] implies that $X$ is also annularly quasiconvex, i.e. there is a constant $\Lambda \geq 1$ such that whenever $B \subset X$ is a ball and $y, z \in B \backslash \frac{1}{2} B$, there is a curve $\gamma \subset \Lambda B \backslash(2 \Lambda)^{-1} B$ connecting $y$ to $z$ and such that $l_{\gamma} \leq \Lambda d(y, z)$.

It follows from Theorem 6.5 in Buckley-Herron-Xie [24] that also the sphericalization $(\widehat{X}, \hat{d})$ is both quasiconvex and annularly quasiconvex. By Proposition 3.1 and Theorem 3.6 in Li-Shanmugalingam [44], $\left(\widehat{X}, \hat{d}, \mu_{a}\right)$ is Ahlfors $Q$-regular and supports a $q$-Poincaré inequality.

Proposition 5.1. The space $(\widehat{X}, \hat{d}, \hat{\mu})$ supports a p-Poincaré inequality and $\hat{\mu}$ is doubling on $(\widehat{X}, \hat{d})$.

Proof. Since $\mu$ is Ahlfors $Q$-regular, we have by (3.2),

$$
\begin{aligned}
d \hat{\mu}(x) & =\frac{d \mu(x)}{(1+d(x, a))^{2 p}} \simeq(1+d(x, a))^{2(Q-p)} \frac{d \mu(x)}{\mu(B(a, 1+d(x, a)))^{2}} \\
& =(1+d(x, a))^{2(Q-p)} d \mu_{a}(x) \simeq \hat{d}(x, \infty)^{2(p-Q)} d \mu_{a}(x) .
\end{aligned}
$$

We have already observed that $\mu_{a}$ is Ahlfors $Q$-regular and supports a $q$-Poincaré inequality on $(\widehat{X}, \hat{d})$. Proposition 2.3 with $\alpha=2(p-Q)$ and $\sigma=Q$ now implies that the weight $\hat{d}(x, \infty)^{2(p-Q)}$ belongs to

- $A_{1}\left(\mu_{a}\right)$ when $-Q<2(p-Q) \leq 0$ (which is equivalent to $Q / 2<p \leq Q$ ),

- $A_{\tau}\left(\mu_{a}\right)$ with $\tau>1$ when $0<2(p-Q)<Q(\tau-1)$, i.e. for $p>Q$ and $\tau>2 p / Q-1$.

By Corollary 2.4, $\hat{\mu}$ is doubling on $(\widehat{X}, \hat{d})$.

Since $\mu_{a}$ supports a $q$-Poincaré inequality on $(\widehat{X}, \hat{d})$, Corollary 2.4 also implies that $\hat{\mu}$ supports a $p$-Poincaré inequality when $Q / 2<p \leq Q$.

For $p>Q$, we first need to use Theorem 1.0.1 in Keith-Zhong [36] to see that $\mu_{a}$ supports a $q^{\prime}$-Poincaré inequality on $(\widehat{X}, \hat{d})$ for some $q^{\prime}<q=p Q /(2 p-Q)$. From this it follows, by Corollary 2.4 , that $\hat{\mu}$ supports a $q^{\prime} \tau$-Poincaré inequality on $(\widehat{X}, \hat{d})$ whenever $\tau>2 p / Q-1$, and thus a $p$-Poincaré inequality, as $p>q^{\prime}(2 p / Q-1)$.

Remark 5.2. The proof of Proposition 5.1 also shows that if $(X, d, \mu)$ supports a $q^{\prime}$-Poincaré inequality for some specific $q^{\prime}<p \leq Q$, then also $(\widehat{X}, \hat{d}, \hat{\mu})$ supports a $q^{\prime}$-Poincaré inequality. For $p>Q$ and $q<p Q /(2 p-Q),(\widehat{X}, \hat{d}, \hat{\mu})$ also supports a $\bar{q}$-Poincaré inequality for some $\bar{q}<p$ (in fact for any $\bar{q}>q^{\prime}(2 p-Q) / Q$, but not necessarily with $\left.\bar{q}=q^{\prime}\right)$. Such assumptions of better Poincaré inequalities are often used in the subsequent theory of $p$-harmonic functions. At the same time, since we assume that $X$ (and thus $\widehat{X}$ ) is complete, the self-improvement result in KeithZhong [36, Theorem 1.0.1] shows that the $p$-Poincaré inequality implies a better $q^{\prime}$-Poincaré inequality for some $q^{\prime}<p$ (but with no good explicit control on $q^{\prime}$ ). 
We are now ready to refine Lemma 4.5.

Lemma 5.3. Let $E \subset \widehat{X}$.

(a) If $\infty \notin E$, then $\widehat{C}_{p}(E)=0$ if and only if $C_{p}(E)=0$.

(b) If $\infty \in E$, then $\widehat{C}_{p}(E)=0$ if and only if $C_{p}(E \backslash\{\infty\})=0$ and $p \geq Q$.

(c) If $p>Q$, then $\widehat{C}_{p}(E)=0$ if and only if $E=\varnothing$ or $E=\{\infty\}$.

Proof. (a) This follows directly from Lemma 4.5.

(b) We need to determine when $\widehat{C}_{p}(\{\infty\})=0$, for which we will use results from Björn-Björn-Lehrbäck [10]. As $\mu_{a}$ is Ahlfors $Q$-regular, it is also reverse-doubling, i.e. there are constants $\theta, \tau>1$ such that

$$
\mu_{a}(B(x, \tau r)) \geq \theta \mu_{a}(B(x, r)) \quad \text { whenever } x \in \widehat{X} \text { and } 0<r \leq \operatorname{diam} \widehat{X} / 2 \tau .
$$

Thus we get the following estimates, using (5.3) and denoting balls with respect to $\widehat{X}$ by $\widehat{B}$,

$$
\begin{aligned}
\hat{\mu}(\widehat{B}(\infty, r)) & \simeq \sum_{j=0}^{\infty}\left(r \tau^{-j}\right)^{2(p-Q)} \mu_{a}\left(\widehat{B}\left(\infty, r \tau^{-j}\right) \backslash \widehat{B}\left(\infty, r \tau^{-j-1}\right)\right) \\
& \simeq \sum_{j=0}^{\infty}\left(r \tau^{-j}\right)^{2(p-Q)}\left(r \tau^{-j}\right)^{Q} \simeq r^{2 p-Q}
\end{aligned}
$$

since $2 p-Q>0$, by (5.1). In the notation of [10], this means that the dimension sets of $(\widehat{X}, \hat{d}, \hat{\mu})$ at $\infty$ satisfy

$$
\underline{S}_{0}^{\widehat{X}}(\infty)=(0,2 p-Q] \quad \text { and } \quad \bar{S}_{0}^{\widehat{X}}(\infty)=[2 p-Q, \infty) .
$$

Thus $\widehat{C}_{p}(\{\infty\})=0$ if and only if $p \leq 2 p-Q$ (which is equivalent to $p \geq Q$ ), by Proposition 1.3 in [10].

(c) Let $x \in X$. Then, following the notation in [10], we have $\bar{S}_{0}^{X}(x)=[Q, \infty)$ since $X$ is Ahlfors $Q$-regular. It thus follows from Proposition 1.3 in [10], or Corollary 5.39 in [9], that $C_{p}(\{x\})>0$. Hence, (c) follows from (a) and (b).

\section{6. $\quad$-harmonic functions on unbounded domains}

Recall the assumptions (5.1) and (5.2) on $p$ and $X$. From now on we also assume that $\Omega \subset X$ is a nonempty open set and we regard $\Omega$ simultaneously as a subset of $(X, d, \mu)$ and of $(\widehat{X}, \hat{d}, \hat{\mu})$.

In this section we apply sphericalization and the results from the earlier sections to obtain results about $p$-harmonic functions and the Dirichlet problem on unbounded sets. Let $\operatorname{Lip}_{c}(\Omega)$ denote the space of Lipschitz functions with compact support in $\Omega$.

Definition 6.1. A function $u \in N_{\mathrm{loc}}^{1, p}(\Omega, d, \mu)$ is a (super)minimizer in $\Omega$ (with respect to $(d, \mu))$ if

$$
\int_{\varphi \neq 0} g_{u}^{p} d \mu \leq \int_{\varphi \neq 0} g_{u+\varphi}^{p} d \mu \text { for all (nonnegative) } \varphi \in \operatorname{Lip}_{c}(\Omega),
$$

where $g_{u}$ and $g_{u+\varphi}$ are the minimal $p$-weak upper gradients of $u$ and $u+\varphi$ with respect to $(d, \mu)$. (Super)minimizers with respect to $(\hat{d}, \hat{\mu})$ are defined analogously. A $p$-harmonic function is a continuous minimizer. 
For various characterizations of (super)minimizers see [9] or Björn [3]. It was shown in Kinnunen-Shanmugalingam [39] that under the assumptions of doubling and a $p$-Poincaré inequality, a minimizer can be modified on a set of zero capacity to obtain a $p$-harmonic function. For a superminimizer $u$, it was shown by KinnunenMartio [38] that its lsc-regularization

$$
u^{*}(x):=\operatorname{ess} \liminf _{y \rightarrow x} u(y)=\lim _{r \rightarrow 0} \underset{B(x, r)}{\operatorname{essinf}} u
$$

is also a superminimizer and $u^{*}=u$ q.e.

We are primarily interested in the Dirichlet (boundary value) problem for $p$ harmonic functions, and the associated boundary regularity. The most general way of treating the Dirichlet problem is to consider Perron solutions, and in order to define them we need superharmonic functions.

Definition 6.2. A function $u: \Omega \rightarrow(-\infty, \infty]$, which is not identically $\infty$ in any component of $\Omega$, is superharmonic if it is lsc-regularized (i.e. $u=u^{*}$ ) and $\min \{u, k\}$ is a superminimizer for every $k \in \mathbf{Z}$.

This is not the traditional definition of superharmonic functions, but it is one of several equivalent characterizations used in various places of the nonlinear literature, cf. Kinnunen-Martio [38, Section 7], Björn [2, Theorem 7.1] or [9, Theorem 9.24].

Our choice of the sphericalization measure $\hat{\mu}$ leads to the following invariance result which will be important for applications in this section.

Theorem 6.3. A function $u: \Omega \rightarrow \mathbf{R}$ is a (super)minimizer in $\Omega$ with respect to $(d, \mu)$ if and only if it is a (super)minimizer in $\Omega$ with respect to $(\hat{d}, \hat{\mu})$.

Similarly, p-harmonicity and superharmonicity are the same in the two situations.

Proof. Proposition 4.4 shows that the spaces $N_{\text {loc }}^{1, p}(\Omega, d, \mu)$ and $N_{\text {loc }}^{1, p}(\Omega, \hat{d}, \hat{\mu})$, appearing in Definition 6.1, coincide. Moreover, since $\Omega \subset X, \varphi \in \operatorname{Lip}_{c}(\Omega, d)$ if and only if $\varphi \in \operatorname{Lip}_{c}(\Omega, \hat{d})$, i.e. the sets of test functions for both notions of superminimizers coincide. Proposition 4.3 implies that

$$
\int_{\varphi \neq 0} g_{u}^{p} d \mu=\int_{\varphi \neq 0} \hat{g}_{u}^{p} d \hat{\mu} \text { and } \int_{\varphi \neq 0} g_{u+\varphi}^{p} d \mu=\int_{\varphi \neq 0} \hat{g}_{u+\varphi}^{p} d \hat{\mu}
$$

where $\hat{g}_{u}$ and $\hat{g}_{u+\varphi}$ are the minimal $p$-weak upper gradients of $u$ and $u+\varphi$ in $\Omega$ with respect to $\hat{d}$, respectively. Taking all this into account shows the equivalence of the two notions of superminimizers.

It now follows directly from the definitions that also the two notions of $p$ harmonicity and superharmonicity (with respect to $(d, \mu)$ and $(\hat{d}, \hat{\mu}))$ are equivalent.

We are now ready to define the Perron solutions. We consider the Dirichlet problem with respect to the boundary $\widehat{\partial} \Omega$ corresponding to $\widehat{X}$, i.e. for unbounded $\Omega \subset X$ we set $\widehat{\partial} \Omega=\partial \Omega \cup\{\infty\}$. This is in accordance with the definitions used in Heinonen-Kilpeläinen-Martio [29] and Hansevi [28].

Definition 6.4. Given $f: \widehat{\partial} \Omega \rightarrow \overline{\mathbf{R}}$, let $\mathcal{U}_{f}$ be the set of all superharmonic functions $u$ on $\Omega$, bounded from below, such that

$$
\liminf _{\Omega \ni y \rightarrow x} u(y) \geq f(x)
$$

for all $x \in \widehat{\partial} \Omega$. The upper Perron solution of $f$ is then defined to be

$$
\bar{P} f(x)=\inf _{u \in \mathcal{U}_{f}} u(x), \quad x \in \Omega,
$$


while the lower Perron solution of $f$ is defined by $\underline{P} f=-\bar{P}(-f)$. If $\bar{P} f=\underline{P} f$ and it is real-valued, then we let $P f:=\bar{P} f$ and $f$ is said to be resolutive with respect to $\Omega$.

Note that in (6.1) the limit can be equivalently taken with respect to $d_{a}, \hat{d}$ or $d$, where in the last case $y \rightarrow \infty$ is interpreted in the obvious way. Thus, by Theorem 6.3 , Perron solutions with respect to $(d, \mu)$ and $(\hat{d}, \hat{\mu})$ are the same.

As $\Omega$ is always bounded as a subset of the sphericalization $\widehat{X}$, we can now use all the results about $p$-harmonic functions on bounded sets for it and they will automatically transfer to $p$-harmonic functions and Perron solutions on $\Omega \subset X$ even for unbounded $\Omega$ (with boundary $\widehat{\partial} \Omega$ ).

For the Perron method on $\widehat{X}$ we need to require that $\widehat{C}_{p}(\widehat{X} \backslash \Omega)>0$, which by Lemma 5.3 happens if and only if $C_{p}(X \backslash \Omega)>0$ or $p<Q$. If $p>Q$ this amounts exactly to requiring that $\Omega \neq X$, by Lemma 5.3 .

So from now on we assume that

$$
\Omega \subset X \text { is unbounded, and that } C_{p}(X \backslash \Omega)>0 \text { or } p<Q \text {. }
$$

In this case we get, using the correspondence above, a rich theory also on $\Omega$ seen as an unbounded open subset of the original space $X$. (When $\widehat{C}_{p}(\widehat{X} \backslash \Omega)=0$, the Perron method gets somewhat pathological, but this is not the right place to dwell upon that.)

First we observe that Theorem 4.1 in Björn-Björn-Shanmugalingam [12] (or $[9$, Theorem 10.10]) shows that the Perron solutions are either identically $\pm \infty$ or $p$-harmonic in each component of $\Omega$, and thus in the latter case provide reasonable candidates for solutions of the Dirichlet problem. Moreover, by Theorem 7.2 in Kinnunen-Martio [38] (or [9, Theorem 9.39]), $\underline{P} f \leq \bar{P} f$ for all $f: \widehat{\partial} \Omega \rightarrow \overline{\mathbf{R}}$.

More importantly, various resolutivity results for bounded domains from BjörnBjörn-Shanmugalingam [12], [13], Hansevi [28] and Björn-Björn-Sjödin [15] transform directly into results for unbounded $\Omega$. In unweighted and weighted $\mathbf{R}^{n}, n \geq 2$, some of these consequences recover old results by Kilpeläinen [37] resp. HeinonenKilpeläinen-Martio [29, Chapter 9], but we also obtain many new results.

Some of the results below were obtained by different methods in Hansevi [28] when the space $X$ is $p$-parabolic (i.e. $p \geq Q$, see Björn-Björn-Lehrbäck [10, Remark 8.7]), or more generally when $\Omega$ is $p$-parabolic (see Definition 4.1 in [28] or Definition 7.10 below), which is satisfied for many unbounded sets also when $p<Q$. The Dirichlet problem on unbounded domains with respect to prime end boundaries has been considered in Estep [27].

Some of the obtained consequences are somewhat technical to describe, and in order to keep the exposition limited we will not go into full generality. To avoid misunderstanding and to make the results accessible for readers not interested in the sphericalization $\widehat{X}$, we formulate them using the capacity and other notions on $X$. It should be fairly straightforward for the interested reader to transform also other results from the above mentioned papers, e.g. those involving a better capacity and generalized boundaries.

Theorem 6.5. Assume that (5.1), (5.2) and (6.2) are satisfied. Let $f \in C(\widehat{\partial} \Omega)$ and assume that $h: \widehat{\partial} \Omega \rightarrow \overline{\mathbf{R}}$ vanishes $C_{p}$-q.e. on $\partial \Omega$. Then the following hold:

(a) If $p<Q$ and $h(\infty)=0$ then both $f$ and $f+h$ are resolutive and $P f=P(f+h)$.

(b) If $p \geq Q$ then both $f$ and $f+h$ are resolutive and $P f=P(f+h)$. Moreover, the requirement (6.1) in the definition of $\underline{P} f$ and $\bar{P} f$ only needs to be satisfied at finite boundary points $x \in \partial \Omega$.

Note that for $p>Q$ the function $h$ in Theorem 6.5 is allowed to be nonzero only at $\infty$, since finite points have positive capacity. 
In unweighted $\mathbf{R}^{n}$ with $p>n / 2, p \neq 2$, this result (as well as the uniqueness result in Theorem 6.6 below) is new, although the resolutivity of $f$ was shown already by Kilpeläinen [37, Theorem 1.10].

Proof. Resolutivity and invariance under the perturbation $h$ follow from [12, Theorem 6.1] (or [9, Theorem 10.22]) and the above discussion. We also need to appeal to Lemma 5.3, which shows that $\{\infty\}$ has zero capacity if and only if $p \geq Q$, and can therefore be disregarded in this case.

To conclude the proof, let $p \geq Q$ and $u$ be a superharmonic function on $\Omega$ bounded from below and such that (6.1) holds for all $x \in \partial \Omega$. Then $u \in \mathcal{U}_{f+\hat{h}}$, where $\hat{h}=-\infty \chi_{\{\infty\}}$, and hence the already proved invariance part shows that

$$
u \geq \bar{P}(f+\hat{h})=\bar{P} f .
$$

Taking infimum over all such $u$ shows that the infimum in the definition of Perron solutions does not get smaller by relaxing (6.1). That it cannot get larger is trivial, since it is taken over a larger class of functions.

The following theorem provides us with a unique solution of the Dirichlet problem on unbounded domains. Note, however, that the point at infinity is regarded as a boundary point even if the usual boundary $\partial \Omega$ is bounded. This additional requirement is necessary if $p<Q$.

Theorem 6.6. Assume that (5.1), (5.2) and (6.2) are satisfied. Let $f \in C(\widehat{\partial} \Omega)$. Then $u=P f$ is the unique bounded $p$-harmonic function $u$ on $\Omega$ such that

$$
\lim _{\Omega \ni y \rightarrow x} u(y)=f(x) \quad \text { for } C_{p} \text {-q.e. } x \in \partial \Omega
$$

and also for $x=\infty$ when $p<Q$.

Proof. This follows directly from [9, Theorem 10.24], together with Lemma 5.3 and the above discussion.

For Newtonian functions, and more generally Dirichlet functions, we obtain the following resolutivity and uniqueness results corresponding to Theorems 6.5 and 6.6.

Theorem 6.7. Assume that (5.1), (5.2) and (6.2) are satisfied. Let $f \in D^{p}(X, d, \mu)$ and assume that $h: \widehat{\partial} \Omega \rightarrow \overline{\mathbf{R}}$ vanishes $C_{p}$-q.e. on $\partial \Omega$. Then the following hold:

(a) If $p<Q, h(\infty)=0$ and $\lim _{y \rightarrow \infty} f(y)=: f(\infty)$ exists $($ in $\overline{\mathbf{R}}$ ), then both $f$ and $f+h$ are resolutive and $P f=P(f+h)$.

(b) If $p \geq Q$, then both $f$ and $f+h$ are resolutive and $P f=P(f+h)$. Moreover, the requirement (6.1) in the definition of $\underline{P} f$ and $\bar{P} f$ only needs to be satisfied at finite boundary points $x \in \partial \Omega$.

Proof. The resolutivity and invariance follow from the sphericalization process together with (the bounded case of) Theorem 7.6 in Hansevi [28], if we can show that $f \in D^{p}(\widehat{X}, \hat{d}, \hat{\mu})$. To this end, note that $f \in D^{p}(X, \hat{d}, \hat{\mu})$, by Proposition 4.3. Let $\hat{g} \in L^{p}(X, \hat{\mu})$ be an upper gradient of $f$ in $X$ with respect to $\hat{d}$, and let $\hat{g}(\infty)$ be arbitrary.

If $p \geq Q$ then $\widehat{C}_{p}(\{\infty\})=0$, by Lemma 5.3 , and hence $p$-almost every curve in $\widehat{X}$ avoids $\infty$ (by [9, Proposition 1.48]), which immediately implies that $\hat{g}$ is a $p$-weak upper gradient of $f$ in $\widehat{X}$, and thus $f \in D^{p}(\widehat{X}, \hat{d}, \hat{\mu})$.

For $p<Q$, let $\gamma \subset \widehat{X}$ be a rectifiable curve. If $\gamma \subset X$, there is nothing to prove. So, by splitting $\gamma$ into parts and reversing the orientation, if necessary, we can assume that $\gamma^{-1}(\{\infty\})=\{0\}$. The continuity of $f$ at $\infty$ then yields

$$
\left|f(\gamma(0))-f\left(\gamma\left(l_{\gamma}\right)\right)\right|=\lim _{t \rightarrow 0}\left|f(\gamma(t))-f\left(\gamma\left(l_{\gamma}\right)\right)\right| \leq \int_{\gamma} \hat{g} d s .
$$


Since $\gamma$ was arbitrary, we conclude that $\hat{g}$ is an upper gradient of $f$ in $\widehat{X}$, and thus $f \in D^{p}(\widehat{X}, \hat{d}, \hat{\mu})$.

The last part in (b) is proved in the same way as the similar statement in Theorem 6.5.

Proposition 6.8. Assume that (5.1), (5.2) and (6.2) are satisfied. Let $f \in D^{p}(X, d, \mu)$ be bounded and assume that $u$ is a bounded $p$-harmonic function in $\Omega$ such that

$$
\lim _{\Omega \ni y \rightarrow x} u(y)=f(x) \quad \text { for } C_{p} \text {-q.e. } x \in \partial \Omega \text {. }
$$

If $p<Q$, assume in addition that $\lim _{\Omega \ni y \rightarrow \infty} u(y)=\lim _{y \rightarrow \infty} f(y)$. Then $u=P f$.

Note that, unlike in Theorem 6.6, the existence of a function satisfying (6.3) for noncontinuous boundary data is not guaranteed by the Kellogg property (Theorem 6.9 below).

Proof. By the proof of Theorem 6.7, $f \in D^{p}(\widehat{X}, \hat{d}, \hat{\mu})$ and hence $f \in N^{1, p}(\widehat{X}, \hat{d}, \hat{\mu})$ since it is bounded. Thus, the statement follows directly from [9, Corollary 10.16], together with the sphericalization process and Lemma 5.3.

Also boundary regularity results carry over to unbounded domains, the most important is maybe the Kellogg property, which we obtain using [11, Theorem 3.9], together with Lemma 5.3. Recall that $x \in \widehat{\partial} \Omega$ is called regular if

$$
\lim _{\Omega \ni y \rightarrow x} P f(y)=f(x) \quad \text { for all } f \in C(\widehat{\partial} \Omega) .
$$

Theorem 6.9. (Kellogg property) Assume that (5.1), (5.2) and (6.2) are satisfied. The set of irregular boundary points in $\partial \Omega$ has $C_{p}$-capacity zero. Moreover, $\infty$ is always regular if $p<Q$.

Useful properties of boundary regularity are its locality and the barrier characterization, which transfer to unbounded domains in the following way. A superharmonic function $u$ in $\Omega$ is a barrier at $x_{0} \in \widehat{\partial} \Omega$ if

$$
\lim _{\Omega \ni y \rightarrow x_{0}} u(y)=0 \quad \text { and } \quad \liminf _{\Omega \ni y \rightarrow x} u(y)>0 \quad \text { for every } x \in \widehat{\partial} \Omega \backslash\left\{x_{0}\right\} .
$$

Theorem 6.10. Assume that (5.1), (5.2) and (6.2) are satisfied. A point $x_{0} \in \widehat{\partial} \Omega$ is regular if and only if there exists a barrier at $x_{0}$. (Equivalently, the barrier can be chosen positive and continuous.) In this case, (6.4) holds for all bounded $f: \widehat{\partial} \Omega \rightarrow \mathbf{R}$ which are continuous at $x_{0}$.

Moreover, regularity is local in the following sense:

(a) A finite boundary point $x_{0} \in \partial \Omega$ is regular with respect to $\Omega$ if and only if it is regular with respect to $\Omega \cap G$ for some (or equivalently all) open $G \ni x_{0}$.

(b) The point $\infty \in \widehat{\partial} \Omega$ is regular with respect to $\Omega$ if and only if it is regular with respect to $\Omega \backslash K$ for some (or equivalently all) compact $K$.

Proof. This follows directly from the sphericalization process, together with Theorems 4.2 and 6.1 in Björn-Björn [7] (or [9, Theorem 11.11]).

Some of the above results are new also for unweighted $\mathbf{R}^{n}, n \geq 2$, with $p>n / 2$, but the Kellogg property and the barrier characterization appeared already in Kilpeläinen [37, Theorems 1.5 and Corollary 5.6] in this setting. We also obtain several new characterizations of boundary regularity in unbounded sets corresponding to the results in Björn-Björn [7, Theorem 6.1] (or [9, Theorem 11.11]), see also Heinonen-Kilpeläinen-Martio [29, Chapter 9]. 
A direct consequence of Theorem 6.10 (a) is that the Wiener type criterion from Björn-MacManus-Shanmugalingam [22] and Björn [20], [21] can be applied also to finite boundary points in unbounded domains. Regularity of the point at $\infty$ will be discussed in the next section.

Other boundary regularity results that generalize from bounded to unbounded sets are the trichotomy classification into regular, semiregular and strongly irregular boundary points from Björn [4] (or [9, Chapter 13]). These results can be applied to finite boundary points as well as to $\infty$. Moreover, the results on approximation by regular sets and on so-called Wiener solutions of the Dirichlet problem from Björn-Björn [8] (or [9, Chapter 14]) generalize in a similar way.

Furthermore, since the $p$-energy is preserved under sphericalization, also quasiminimizers are preserved in just the same way as $p$-harmonic functions. We can thus generalize many earlier boundary regularity results for quasiminimizers from bounded to unbounded sets (provided that $X$ satisfies our standing assumptions). These include results in [3, Sections 4 and 5], [4, Section 6], [5, Section 7], [8, Theorem 1.1], [16, Section 6], [18, Theorems 2.12 and 2.13] and [21, Theorem 1.1].

\section{Resolutivity and regularity at $\infty$}

For unbounded $\Omega$, what happens at $\infty$ is of particular interest. Recall that by Theorem 6.9, $\infty$ is always regular if $p<Q$. Theorem $6.10(\mathrm{~b})$, combined with Theorem 7.5 in Björn [5] (or [9, Theorem 11.27]), immediately implies the following result.

Proposition 7.1. Assume that (5.1), (5.2) and (6.2) are satisfied. Let $k>0$. Then $\infty \in \widehat{\partial} \Omega$ is regular with respect to $\Omega$ if and only if it is regular with respect to every unbounded component $G$ of $\Omega \backslash \overline{B(a, k)}$. This in particular guarantees regularity of the point at $\infty$ if there are no such unbounded components.

A simple application of the last part of Proposition 7.1 is demonstrated in the following example.

Example 7.2. The point $\infty \in \partial \Omega$ is regular with respect to

$$
\Omega=(0,1)^{2} \cup \bigcup_{j=1}^{\infty}\left(2^{-j}, 2^{1-j}\right) \times\left(0,2^{j}\right)
$$

since $\Omega \backslash \overline{B(0,2)}$ consists only of bounded components.

The capacity $\widehat{C}_{p}$ (or rather its variational analogue cap ${ }_{p}^{\widehat{X}}$ ) near $\infty$ plays an important role here through Wiener type criteria. Using (4.1), $\operatorname{cap}_{p}^{\widehat{X}}$ can be described by means of functions on $X$. This makes it possible to rewrite the Wiener type integrals at $\infty$, appearing in Björn [20], [21] and Björn-MacManus-Shanmugalingam [22], in terms of $\Omega$ and $X$. For a more hands-on result we use the fact that porosity is sufficient for boundary regularity and formulate the following practical condition.

Theorem 7.3. Assume that (5.1), (5.2) and (6.2) are satisfied. Assume that for some $k>0$ and for each unbounded component $G$ of $\Omega \backslash \overline{B(a, k)}$ there exist $\theta>0$ and $x_{j} \in X$ such that $d\left(x_{j}, a\right) \rightarrow \infty$ as $j \rightarrow \infty$ and

$$
B\left(x_{j}, \theta d\left(x_{j}, a\right)\right) \cap G=\varnothing \quad \text { for all } j=1,2, \ldots
$$

Then $\infty \in \widehat{\partial} \Omega$ is regular. 
Proof. Simple geometrical considerations show that (7.1) implies the existence of $\theta^{\prime}>0$ so that for sufficiently large $j$ (and with $\widehat{B}$ denoting balls with respect to $\widehat{X}$ ),

$$
\widehat{B}\left(x_{j}, \theta^{\prime} \hat{d}\left(x_{j}, \infty\right)\right) \subset \widehat{B}\left(\infty, 2 \hat{d}\left(x_{j}, \infty\right)\right) \backslash G,
$$

i.e. that $G$ is porous at $\infty$ with respect to $\hat{d}$. The sphericalization argument together with [9, Corollary 11.25 (c)] then implies that $\infty \in \widehat{\partial} G$ is regular with respect to the component $G$. Since this is true for every unbounded component of $\Omega \backslash \overline{B(a, k)}$, Proposition 7.1 concludes the proof.

Remark 7.4. The proof of Theorem 7.3 shows that if (7.1) holds for one particular unbounded component of $\Omega \backslash \overline{B(a, k)}$ then $\infty$ is regular with respect to that component.

In order to capture the behaviour of functions from different directions at $\infty$, we will consider the Mazurkiewicz metric $\hat{d}_{M}$ on $\Omega$, generated by $\hat{d}$, cf. Björn-BjörnShanmugalingam [13], [14]. For simplicity we will restrict ourselves to the case when $\Omega$ is connected and satisfies (6.2). For $x, y \in \Omega$, let

$$
\hat{d}_{M}(x, y)=\inf _{E} \operatorname{diam}_{\hat{d}} E,
$$

where the infimum is taken over all connected sets $E \subset \Omega$ containing both $x$ and $y$, and the diameter is taken with respect to $\hat{d}$. It also gives rise to the Mazurkiewicz boundary $\widehat{\partial}_{M} \Omega$ with respect to $\hat{d}_{M}$ in the usual way through completion. The Mazurkiewicz metric $d_{M}$ on $\Omega$ generated by $d$, and the corresponding boundary $\partial_{M} \Omega$, are defined similarly.

It was shown in [13] that upper gradients, Newtonian spaces and $p$-(super)harmonic functions within $\Omega$ are the same with respect to the Mazurkiewicz metric and the original metric generating it. The only change needed in the definition of Perron solutions with respect to the Mazurkiewicz boundary is that the lim inf in (6.1) is with respect to $d_{M}$ and is required on $\widehat{\partial}_{M} \Omega$. To be able to use the results from [13] we assume that $\Omega$ is finitely connected at the boundary with respect to $\hat{d}$, cf. Näkki [46], Väisälä [49], as well as [13] and [14]. This is equivalent to requiring the following two conditions:

(i) $\Omega$ is finitely connected at every finite $x \in \partial \Omega$, i.e. for every $r>0$ and every $x \in \partial \Omega$ there is an open neighbourhood $U \subset B(x, r)$ of $x$ such that $\Omega \cap U$ consists of only finitely many components;

(ii) for every $k>0$ there is a compact set $K \supset \overline{B(a, k)}$ such that $\Omega \backslash K$ has only finitely many components.

By Proposition 2.5 in [14], the conditions (i) and (ii) can equivalently be stated as follows. For $x \in \partial \Omega$ and $r>0$, let $N(r, x)$ be the number of components $V$ of $B(x, r) \cap \Omega$ such that $x \in \bar{V}$, and let $H(r, x)$ be the union of all the other components of $B(x, r) \cap \Omega$. Similarly, let $N(r, \infty)$ be the number of unbounded components of $\Omega \backslash \overline{B(a, r)}$, and let $H(r, \infty)$ be the union of all the bounded components of $\Omega \backslash \overline{B(a, r)}$. Then (i) and (ii) are equivalent to the following two conditions, respectively:

$\left(\mathrm{i}^{\prime}\right) \quad N(r, x)<\infty$ and $x \notin \overline{H(r, x)}$, for every $x \in \partial \Omega$ and $0<r<1$;

(ii') $N(r, \infty)<\infty$ and $H(r, \infty)$ is bounded for every $r>1$.

The condition (ii), or equivalently (ii'), means that the Mazurkiewicz metric $\hat{d}_{M}$ distinguishes between different copies of $\infty$, each corresponding to a decreasing sequence of unbounded components of $\Omega \backslash \overline{B(a, k)}, k=1,2, \ldots$. For unbounded $\Omega \subset \mathbf{R}^{n}$ with sufficiently smooth boundary it is only this requirement at $\infty$ that takes effect since finite connectedness at finite boundary points is automatically satisfied for such smooth domains. 
When discussing the Dirichlet problem with respect to the Mazurkiewicz boundary $\widehat{\partial}_{M} \Omega$ we will restrict ourselves to $f \in C\left(\widehat{\partial}_{M} \Omega\right)$, which is in fact equivalent to $f \in C\left(\partial_{M} \Omega\right)$ together with the requirement that

$\lim _{\partial_{M} \ni x \rightarrow \infty} f(x)$ exists and is finite along each decreasing sequence $\left\{\Omega_{k}\right\}_{k=1}^{\infty}$

of unbounded components $\Omega_{k} \subset \Omega \backslash \overline{B(a, k)}, k=1,2, \ldots$. Here we say that $X \ni$ $x_{n} \rightarrow \infty$, as $n \rightarrow \infty$, along such a sequence $\Omega_{1} \supset \Omega_{2} \supset \ldots$ if for every $k$ there exists $N_{k}$ such that all $x_{n}$ with $n \geq N_{k}$ belong to the $d_{M}$-closure of $\Omega_{k}$. Note that the limit is allowed to be different for different directions towards $\infty$, i.e. for different sequences $\left\{\Omega_{k}\right\}_{k=1}^{\infty}$.

Under the assumption of finite connectedness at the boundary, it can be verified that $\widehat{\partial}_{M} \Omega$ equals $\partial_{M} \Omega$, together with all the copies of $\infty$ from different directions. Finiteness at the boundary is equivalent to the compactness of the $\hat{d}_{M}$-closure of $\Omega$, see Björn-Björn-Shanmugalingam [14, Theorem 1.1] or Karmazin [35, Theorem 1.3.8]. In the terminology of Adamowicz-Björn-Björn-Shanmugalingam [1] and Estep [27], the sequence $\left\{\Omega_{k}\right\}_{k=1}^{\infty}$ can be identified with a so-called prime end at $\infty$, see [1, Theorem 9.6 and Corollary 10.9].

The following example shows that there can be uncountably many such directional sequences towards $\infty$.

Example 7.5. Let

$$
A=\left\{\alpha=0 . \alpha_{1} \ldots \alpha_{n} \in(0,1): \alpha_{j} \in\{0,1\}, n=1,2, \ldots\right\}
$$

be the set of all $\alpha \in(0,1)$ with finite binary expansions. Let $\|\alpha\|$ denote the last nonzero position. For each $\alpha \in A$, consider the ray

$$
F_{a}=\left\{z=r e^{i \alpha \pi} \in \mathbf{C}: r \geq\|\alpha\|\right\}
$$

and let $\Omega$ be the upper half-plane with all these rays $F_{\alpha}, \alpha \in A$, removed. Then $\Omega$ is finitely connected at the boundary and there are uncountably many directions towards $\infty$ within $\Omega$, corresponding to each $\alpha \in(0,1) \backslash A$. Theorem 7.3 implies that the point at $\infty$ is regular with respect to $\Omega$.

The following result is a direct consequence of the sphericalization process and Theorem 8.2 in Björn-Björn-Shanmugalingam [13].

Theorem 7.6. Assume that $\Omega$ satisfies (i) and (ii), and that (5.1), (5.2) and (6.2) are satisfied. Let $f \in C\left(\partial_{M} \Omega\right)$ be such that $\lim _{\partial_{M} \Omega \ni x \rightarrow \infty} f(x)$ exists and is finite along each decreasing sequence of unbounded components of $\Omega \backslash \overline{B(a, k)}, k=1,2, \ldots$, in the sense of (7.2). Then $f$ is resolutive with respect to the Mazurkiewicz boundary.

Here, and in Theorem 7.7, it is assumed that $f$ takes the value at a boundary point at infinity given by the above limit, which may depend on the direction towards $\infty$.

Boundary regularity with respect to the Mazurkiewicz boundary in bounded domains that are finitely connected at the boundary was studied in Björn [6]. The results therein can therefore be reformulated using sphericalization for unbounded domains as well. We leave the details to the interested reader and restrict ourselves to the following special case which can be combined with the conditions in Proposition 7.1, Theorem 7.3 and Remark 7.4.

Theorem 7.7. Let $\Omega$ and $f$ be as in Theorem 7.6. Assume that

$$
\lim _{\partial_{M} \Omega \ni x \rightarrow \infty} f(x)=A \in \mathbf{R} \quad \text { along a decreasing sequence }\left\{\Omega_{k}\right\}_{k=1}^{\infty}
$$


of unbounded components $\Omega_{k} \subset \Omega \backslash \overline{B(a, k)}, k=1,2, \ldots$ If $\infty$ is regular with respect to $\Omega_{j}$ for some $j$, then the Perron solution $P_{\Omega}^{M} f$ in $\Omega$ with respect to the Mazurkiewicz boundary satisfies

$$
\lim _{\Omega \ni x \rightarrow \infty} P_{\Omega}^{M} f(x)=A \quad \text { along }\left\{\Omega_{k}\right\}_{k=1}^{\infty},
$$

i.e. the point at infinity in the direction of $\left\{\Omega_{k}\right\}_{k=1}^{\infty}$ is regular.

Proof. Let $\varepsilon>0$. It is easily verified using (7.3) that there exists $k \geq j$ such that $|f-A|<\varepsilon$ on $\partial_{M} \Omega_{k} \cap \partial_{M} \Omega$. By Theorem 7.6, $f$ is resolutive with respect to the Mazurkiewicz boundary. It then follows from the definition of Perron solutions with respect to $\partial_{M} \Omega$ and $\partial_{M} \Omega_{k}$ that

$$
P_{\Omega}^{M} f=P_{\Omega_{k}}^{M} f_{k} \quad \text { in } \Omega_{k}, \quad \text { where } f_{k}= \begin{cases}f & \text { on } \partial_{M} \Omega_{k} \cap \partial_{M} \Omega, \\ P_{\Omega}^{M} f & \text { on } \partial_{M} \Omega_{k} \cap \Omega,\end{cases}
$$

cf. Lemma 3.3 in Björn [19]. Let

$$
\tilde{f}_{k}= \begin{cases}A+\varepsilon & \text { on } \partial \Omega_{k} \cap \partial \Omega \\ P_{\Omega}^{M} f & \text { on } \partial \Omega_{k} \cap \Omega .\end{cases}
$$

Then it is easy to see that $P_{\Omega_{k}}^{M} f_{k} \leq \bar{P}_{\Omega_{k}} \tilde{f}_{k}$ in $\Omega_{k}$. By Corollary 4.4 in BjörnBjörn [7] (or [9, Corollary 11.3]), $\infty$ is regular with respect to $\Omega_{k} \subset \Omega_{j}$. Since $\partial \Omega_{k} \cap \Omega \subset \partial B(a, k)$, we see that $\tilde{f}_{k}$ is continuous at $\infty \in \widehat{\partial} \Omega_{k}$, and thus we obtain using [7, Theorem 6.1] (or [9, Theorem 11.11]) that

$$
\limsup _{\Omega \ni x \rightarrow \infty} P_{\Omega}^{M} f(x) \leq \limsup _{\Omega \ni x \rightarrow \infty} \bar{P}_{\Omega_{k}} \tilde{f}_{k}(x) \leq A+\varepsilon \quad \text { along }\left\{\Omega_{k}\right\}_{k=1}^{\infty} .
$$

Letting $\varepsilon \rightarrow 0$ and applying the same argument to $-f$ concludes the proof.

Example 7.8. For $j=0,1,2, \ldots$, let

$$
F_{j}=\left\{x=\left(x_{1}, x_{2}\right) \in \mathbf{R}^{2}: x_{2}=2^{j} x_{1} \geq 2^{j}\right\}
$$

and $\Omega=(0, \infty)^{2} \backslash \bigcup_{j=0}^{\infty} F_{j}$. Then $\Omega$ is finitely connected at the boundary and the "fingers" of $\Omega$ (each between $F_{j}$ and $F_{j+1}$ ) determine countably many directions towards $\infty$, accumulating towards the strip $(0,1) \times(0, \infty) \subset \mathbf{R}^{2}$, which also determines one direction towards $\infty$. By Theorem 7.3, $\infty \in \widehat{\partial} \Omega$ is regular with respect to $\Omega$, and so are all the $\widehat{\partial}_{M} \Omega$-boundary points at infinity, by Theorem 7.7 .

Example 7.9. For $j=0,1,2, \ldots$, let

$$
F_{j}^{\prime}=\left\{x=\left(x_{1}, x_{2}\right) \in \mathbf{R}^{2}: x_{2}=2^{j} x_{1} \geq 1\right\}
$$

and $\Omega^{\prime}=(0, \infty)^{2} \backslash \bigcup_{j=0}^{\infty} F_{j}^{\prime}$. The "fingers" of $\Omega$ (each between $F_{j}^{\prime}$ and $F_{j+1}^{\prime}$ ) determine countably many directions towards $\infty$, accumulating towards the positive $x_{2}$-axis. This sequence of "fingers" also determines one direction towards $\infty$ even though there is no single finger corresponding to it.

Since $\Omega$ is not finitely connected at the boundary, Theorems 7.6, 7.7 and 7.11 are not applicable. Nevertheless, Theorem 7.3 shows that the point at $\infty$ is regular with respect to $\Omega$.

The influence of each of the directions to infinity on the Dirichlet problem is determined by the capacity $\widehat{C}_{p}^{M}$, which is adapted to $\Omega$ and the Mazurkiewicz metric, as in Björn-Björn-Shanmugalingam [13]. To keep the exposition simple, we will restrict ourselves to the following sufficient condition guaranteeing that a point at infinity is negligible along a decreasing sequence $\Omega_{1} \supset \Omega_{2} \supset$... of unbounded components of $\Omega \backslash \overline{B(a, k)}, k=1,2, \ldots$, cf. Definition 4.1 in Hansevi [28]. 
Definition 7.10. We say that a decreasing sequence $\left\{\Omega_{k}\right\}_{k=1}^{\infty}$ of unbounded components of $\Omega \backslash \overline{B(a, k)}, k=1,2, \ldots$, is $p$-parabolic towards $\infty$ if there exist $u_{j} \in N^{1, p}(\Omega)$ satisfying $u_{j}=0$ in $\Omega \cap B(a, j)$,

$$
\int_{\Omega_{j}} g_{u_{j}}^{p} d \mu \rightarrow 0, \quad \text { as } j \rightarrow \infty
$$

and $\liminf \operatorname{in}_{x \rightarrow \infty} u_{j}(x) \geq 1$ along $\left\{\Omega_{k}\right\}_{k=1}^{\infty}$ for each $j=1,2, \ldots$.

Theorem 7.11. Let $\Omega$ and $f$ be as in Theorem 7.6. If $\left\{\Omega_{k}\right\}_{k=1}^{\infty}$ is p-parabolic towards $\infty$ then the point at $\infty$ is negligible for the Perron solution Pf along $\left\{\Omega_{k}\right\}_{k=1}^{\infty}$, i.e. the requirement $\lim _{\inf _{\Omega \ni x \rightarrow \infty}} u(x) \geq f(\infty)$ in the definition of $\bar{P} f$ does not need to be satisfied when $x \rightarrow \infty$ along $\left\{\Omega_{k}\right\}_{k=1}^{\infty}$.

Proof. This follows from Theorem 8.2 in Björn-Björn-Shanmugalingam [13] and the fact that (7.4), together with (4.1), implies that the corresponding point in the Mazurkiewicz boundary $\widehat{\partial}_{M} \Omega$ has zero $\widehat{C}_{p}^{M}$-capacity. See the proof of Theorem 6.5 for further details.

Note that if, as in Example 7.5, there are uncountably many directions towards $\infty$, it may happen that $\widehat{C}_{p}^{M}(E, \Omega)>0$, even if each direction towards $\infty$ is $p$ parabolic, where $E$ is the set consisting of all the $p$-parabolic directions towards $\infty$. Thus we cannot conclude that all of $E$ can be ignored in the definition of Perron solutions, at least not using the technique here.

\section{8. $p$-harmonic measure is nonadditive on null sets}

The $p$-harmonic measure of a set $E \subset \partial \Omega$ is $\omega_{p}(E ; \Omega):=\bar{P} \chi_{E}$, where $\chi_{E}$ is the characteristic function of $E$. When $p=2$ it becomes the usual (upper) harmonic measure.

For the upper half plane $\mathbf{R}_{+}^{2}=\left\{(x, y) \in \mathbf{R}^{2}: y>0\right\}$, equipped with the Lebesgue measure $m$, Llorente-Manfredi-Wu [45] showed that for any $p \neq 2$ there are sets $E_{1}, \ldots, E_{k}$ such that $\bigcup_{j=1}^{k} E_{j}=\mathbf{R}$, but $\omega_{p}\left(E_{j} ; \mathbf{R}_{+}^{2}\right)=m\left(\mathbf{R} \backslash E_{j}\right)=0$, $j=1, \ldots, k$. Since $\omega_{p}\left(\mathbf{R} ; \mathbf{R}_{+}^{2}\right)=1$, it follows that there are two sets $A_{1}, A_{2} \subset \mathbf{R}$ such that

$$
\omega_{p}\left(A_{1} ; \mathbf{R}_{+}^{2}\right)=\omega_{p}\left(A_{2} ; \mathbf{R}_{+}^{2}\right)=0<\omega_{p}\left(A_{1} \cup A_{2} ; \mathbf{R}_{+}^{2}\right),
$$

showing that the $p$-harmonic measure is not finitely subadditive on zero sets. As in Definition 6.4 and [29, Section 11], the $p$-harmonic measure in [45] is taken with respect to the compactified boundary $\widehat{\partial} \mathbf{R}_{+}^{2}=\mathbf{R} \cup\{\infty\}$. The equality $\omega_{p}\left(\mathbf{R} ; \mathbf{R}_{+}^{2}\right)=$ $\omega_{p}\left(\widehat{\partial} \mathbf{R}_{+}^{2} ; \mathbf{R}_{+}^{2}\right)=1$ then follows from [29, Theorem 11.4] and the following lemma. The lemma was mentioned in [45], but for the reader's convenience we provide a proof.

Lemma 8.1. Let $X=\mathbf{R}^{n}, n \geq 2$, equipped with the Lebesgue measure, and let $p>1$. Then $\omega_{p}\left(\{\infty\} ; \mathbf{R}_{+}^{n}\right)=0$.

Proof. Let $f=\chi_{\{\infty\}}$. Consider first the case when $1<p<n$. Let

$$
u_{k}(x)=1-\left(\frac{|x-(0, \ldots, 0,-k)|}{k}\right)^{(p-n) /(p-1)}, \quad x \in \mathbf{R}_{+}^{n} .
$$

Then $u_{k} \in \mathcal{U}_{f}$ and thus for each $x=\left(x_{1}, \ldots, x_{n}\right) \in \mathbf{R}_{+}^{n}$,

$$
\omega_{p}\left(\{\infty\} ; \mathbf{R}_{+}^{n}\right)(x) \leq u_{k}(x) \leq 1-\left|\frac{x_{n}+k}{k}\right|^{(p-n) /(p-1)} \rightarrow 0, \quad \text { as } k \rightarrow \infty .
$$


For $p \geq n, k \geq 2$ and $x \in \mathbf{R}_{+}^{n}$, we instead let

$$
u_{k}(x)= \begin{cases}\frac{1}{k}|x|^{(p-n) /(p-1)}, & \text { if } p>n, \\ \log \frac{|x-(0, \ldots, 0,-k)|}{k}, & \text { if } p=n .\end{cases}
$$

These are estimated similarly.

The proof in [45] uses an idea of Wolff [50] which involves intricate use of scaling and translation invariance, and is thus not applicable to bounded domains. Now we are able to construct similar bounded examples using sphericalization. As mentioned in [45], by adding dummy variables one directly obtains similar examples also on $\mathbf{R}_{+}^{n}, n \geq 3$. Using the sphericalization technique in this paper we obtain the following example on the sphere $\mathbf{S}^{n}$.

Example 8.2. Let $X=\mathbf{R}^{n}$ and $\widehat{X}=\mathbf{S}^{n}$ be its sphericalization, $n \geq 2,1<p<\infty$, $p \neq 2, \Omega=\mathbf{R}_{+}^{n}$, and $A_{1}$ and $A_{2}$ be as above. When $\Omega$ is seen as a bounded subdomain of $\widehat{X}$, we have that

$$
\omega_{p}\left(A_{1} ; \Omega\right)=\omega_{p}\left(A_{2} ; \Omega\right)=0<\omega_{p}\left(A_{1} \cup A_{2} ; \Omega\right),
$$

showing that also in this situation the $p$-harmonic measure is not finitely subadditive on zero sets. The sets $E_{j}$ transfer similarly.

Note that the metric $\hat{d}$ from (3.1), that we equip the sphere $\widehat{X}=\mathbf{S}^{n}$ with, is not the usual spherical (inner) metric, nor the metric induced by $\mathbf{R}^{n+1}$. Moreover, $\widehat{X}=\mathbf{S}^{n}$ is equipped with a measure which depends on $p$.

Just as sphericalization can be used to map an unbounded space into a bounded space, flattening can be used in the converse direction, see Li-Shanmugalingam [44] and Durand-Cartagena-Li [25], [26]. Thus we can flatten the sphere in Example 8.2, mapping a point $c \notin \bar{\Omega}$ to infinity, e.g. $c=(0, \ldots, 0,-1)$. This would produce an example of a bounded domain in $\mathbf{R}^{n}$ in the spirit of the example by LlorenteManfredi-Wu [45], where $\mathbf{R}^{n}$ necessarily needs to be equipped with some weighted measure, if $p \neq n$.

In this particular case, we can obtain the same result by using spherical inversion as follows: Let $X=\mathbf{R}^{n}$, equipped with the Lebesgue measure $d x$, and let $Y$ be another copy of $\mathbf{R}^{n}$. Let $\Phi: X \backslash\{0\} \rightarrow Y \backslash\{0\}$ be given by

$$
\Phi(x)=\frac{x}{|x|^{2}}, \quad \text { and thus } \quad \Phi^{-1}(y)=\frac{y}{|y|^{2}}
$$

We extend $\Phi$ so that $\Phi(\infty)=0$ and $\Phi(0)=\infty$. As we shall see, to preserve the $p$-energy we need to equip $Y$ with the measure $d \hat{\mu}(y)=|y|^{2(p-n)} d y$.

Let $\Omega \subset X$ be open and consider a function $u: \Omega \rightarrow \overline{\mathbf{R}}$. Let $\widehat{\Omega}=\Phi(\Omega)$ and $\hat{u}=u \circ \Phi^{-1}: \widehat{\Omega} \rightarrow \overline{\mathbf{R}}$. As in Proposition 4.3 we see that

$$
\hat{g}_{\hat{u}}(y)=|x|^{2} g_{u}(x)=\frac{g_{u}(x)}{|y|^{2}}, \quad \text { where } y=\Phi(x),
$$

provided that $u \in D_{\mathrm{loc}}^{p}(\Omega)$ and $\hat{u} \in D_{\mathrm{loc}}^{p}(\widehat{\Omega})$. It then follows that

$$
\int_{\widehat{\Omega}} \hat{g}_{\hat{u}}^{p} d \hat{\mu}(y)=\int_{\Omega}\left(|x|^{2} g_{u}(x)\right)^{p}|x|^{2(n-p)}|x|^{-2 n} d x=\int_{\Omega} g_{u}^{p} d x
$$

i.e. the $p$-energy is indeed preserved (and $u \in D_{\text {loc }}^{p}(\Omega)$ if and only if $\hat{u} \in D_{\text {loc }}^{p}(\widehat{\Omega})$ ). 
Example 8.3. To get a bounded example in the spirit of Llorente-Manfredi$\mathrm{Wu}$ [45], we first shift their example and let $\Omega=\left\{\left(x_{1}, \ldots, x_{n}\right): x_{n}>\frac{1}{2}\right\}$. (Their sets $E_{j}$ and $A_{j}$ should also be shifted using the map $x \mapsto x+\left(0, \ldots, 0, \frac{1}{2}\right)$.) After performing the inversion (8.1), we directly get a bounded example, with $\widehat{\Omega}$ being the unit ball in $\mathbf{R}^{n}$ centred at $(0, \ldots, 0,1)$. The sets $E_{j}$ and $A_{j}$ transfer as described above into $\widehat{E}_{j}=\Phi\left(E_{j}\right), \hat{A}_{j}=\Phi\left(A_{j}\right) \subset \partial \widehat{\Omega}$.

To fit within the standard framework as e.g. in Heinonen-Kilpeläinen-Martio [29], we need $\hat{\mu}$ to be a $p$-admissible measure. By [29, p. 10], this happens if and only if $2(p-n)>-n$, i.e. $p>n / 2$, recovering a condition that we have encountered earlier. (If $2(p-n) \leq-n$ then the weight is not locally integrable around the origin and thus cannot be $p$-admissible.)

When $p=n>2$ the weight is 1 and thus $\left(\mathbf{R}^{n}, d \hat{\mu}\right)$ is the unweighted $\mathbf{R}^{n}$.

\section{References}

1. Adamowicz, T., Buörn, A., Buörn, J. and Shanmugalingam, N., Prime ends for domains in metric spaces, Adv. Math. 238 (2013), 459-505. 18

2. BJÖRn, A., Characterizations of $p$-superharmonic functions on metric spaces, Studia Math. 169 (2005), 45-62. 12

3. BJörn, A., A weak Kellogg property for quasiminimizers, Comment. Math. Helv. 81 (2006), 809-825. 12, 16

4. BJöRN, A., A regularity classification of boundary points for $p$-harmonic functions and quasiminimizers, J. Math. Anal. Appl. 338 (2008), 39-47. 16

5. BJörn, A., Cluster sets for Sobolev functions and quasiminimizers, J. Anal. Math. 112 (2010), 49-77. 16

6. BJÖRN, A., The Kellogg property and boundary regularity for $p$-harmonic functions with respect to the Mazurkiewicz boundary and other compactifications, to appear in Complex Var. Elliptic Equ. doi: 10.1080/17476933.2017.1410799. 18

7. BJÖRN, A. and BJÖRN, J., Boundary regularity for $p$-harmonic functions and solutions of the obstacle problem, J. Math. Soc. Japan 58 (2006), 1211-1232. $2,3,15,19$

8. BJÖRN, A. and BJÖRN, J., Approximations by regular sets and Wiener solutions in metric spaces, Comment. Math. Univ. Carolin. 48 (2007), 343-355. 16

9. BJÖRn, A. and BJörn, J., Nonlinear Potential Theory on Metric Spaces, EMS Tracts in Mathematics 17, European Math. Soc., Zürich, 2011. 4, 7, 9, 10, 11, $12,13,14,15,16,17,19$

10. BJörn, A., BJörn, J. and LehrBÄCK, J., Sharp capacity estimates for annuli in weighted $\mathbf{R}^{n}$ and metric spaces, Math. Z. 286 (2017), 1173-1215. 11, 13

11. Björn, A., Bü̈rn, J. and Shanmugalingam, N., The Dirichlet problem for $p$-harmonic functions on metric spaces, J. Reine Angew. Math. 556 (2003), 173-203. 2, 15

12. Björn, A., Buörn, J. and Shanmugalingam, N., The Perron method for p-harmonic functions in metric spaces, J. Differential Equations 195 (2003), 398-429. 2, 13, 14

13. Björn, A., Buörn, J. and Shanmugalingam, N., The Dirichlet problem for $p$-harmonic functions with respect to the Mazurkiewicz boundary, and new capacities, J. Differential Equations 259 (2015), 3078-3114. 3, 13, 17, 18, 19, 20

14. Buörn, A., Buörn, J. and Shanmugalingam, N., The Mazurkiewicz distance and sets that are finitely connected at the boundary, J. Geom. Anal. 26 (2016), 873-897. 17, 18 
15. BJörn, A., Buörn, J. and SJöDin, T., The Dirichlet problem for $p$-harmonic functions with respect to arbitrary compactifications, Rev. Mat. Iberoam. 34 (2018), 1323-1360. 13

16. Bü̈rn, A. and Martio, O., Pasting lemmas and characterizations of boundary regularity for quasiminimizers, Results Math. 55 (2009), 265-279. 16

17. BJÖRn, J., Poincaré inequalities for powers and products of admissible weights, Ann. Acad. Sci. Fenn. Math. 26 (2001), 175-188. 6

18. BJöRN, J., Boundary continuity for quasiminimizers on metric spaces, Illinois J. Math. 46 (2002), 383-403. 2, 16

19. Björn, J., Wiener criterion for Cheeger $p$-harmonic functions on metric spaces, in Potential Theory in Matsue, Adv. Stud. Pure Math. 44, pp. 103-115, Mathematical Society of Japan, Tokyo, 2006. 19

20. BJörn, J., Fine continuity on metric spaces, Manuscripta Math. 125 (2008), 369-381. 16

21. BJÖRN, J., Necessity of a Wiener type condition for boundary regularity of quasiminimizers and nonlinear elliptic equations, Calc. Var. Partial Differential Equations 35 (2009), 481-496. 16

22. Buörn, J., Macmanus, P. and Shanmugalingam, N., Fat sets and pointwise boundary estimates for $p$-harmonic functions in metric spaces, J. Anal. Math. 85 (2001), 339-369. 2, 16

23. Bonk, M. and Kleiner, B., Rigidity for quasi-Möbius group actions, J. Differential Geom. 61 (2002), 81-106. 6

24. Buckley, S. M., Herron, D. and Xie, X., Metric space inversions, quasihyperbolic distance, and uniform spaces, Indiana Univ. Math. J. 57 (2008), 837-890. 6, 10

25. Durand-Cartagena, E. and Li, X., Preservation of $p$-Poincaré inequality for large $p$ under sphericalization and flattening, Illinois J. Math. 59 (2015), 1043-1069. 21

26. Durand-Cartagena, E. and Li, X., Preservation of bounded geometry under sphericalization and flattening: quasiconvexity and $\infty$-Poincaré inequality, Ann. Acad. Sci. Fenn. Math. 42 (2017), 303-324. 21

27. Estep, D., Prime End Boundaries of Domains in Metric Spaces, and the Dirichlet Problem, Ph.D. thesis, University of Cincinnati, Cincinnati, OH, 2015. 13,18

28. Hansevi, D., The Perron method for $p$-harmonic functions in unbounded sets in $\mathbf{R}^{n}$ and metric spaces, Math. Z. 288 (2018), 55-74. 2, 3, 12, 13, 14, 19

29. Heinonen, J., Kilpeläinen, T. and Martio, O., Nonlinear Potential Theory of Degenerate Elliptic Equations, 2nd ed., Dover, Mineola, NY, 2006. 3, 12, 13, $15,20,22$

30. Heinonen, J. and Koskela, P., Quasiconformal maps in metric spaces with controlled geometry, Acta Math. 181 (1998), 1-61. 4

31. Heinonen, J., Koskela, P., Shanmugalingam, N. and Tyson, J. T., Sobolev Spaces on Metric Measure Spaces, New Mathematical Monographs 27, Cambridge Univ. Press, Cambridge, 2015. 4

32. Holopainen, I., Asymptotic Dirichlet problem for the $p$-Laplacian on CartanHadamard manifolds, Proc. Amer. Math. Soc. 130 (2002), 3393-3400. 2

33. Holopainen, I., LAng, U. and VÄHÄKAngas, A., Dirichlet problem at infinity on Gromov hyperbolic metric measure spaces, Math. Ann. 339 (2007), 101-134. 2

34. Holopainen, I. and VÄHÄKangas, A., Asymptotic Dirichlet problem on negatively curved spaces, J. Anal. 15 (2007), 63-110. 2

35. Karmazin, A. P., Quasiisometries, the Theory of Prime Ends and Metric Structures on Domains, Surgut, 2008 (Russian). 18 
36. KeIth, S. and Zhong, X., The Poincaré inequality is an open ended condition, Ann. of Math. 167 (2008), 575-599. 10

37. Kilpeläinen, T., Potential theory for supersolutions of degenerate elliptic equations, Indiana Univ. Math. J. 38 (1989), 253-275. 3, 13, 14, 15

38. Kinnunen, J. and Martio, O., Nonlinear potential theory on metric spaces, Illinois Math. J. 46 (2002), 857-883. 12, 13

39. Kinnunen, J. and Shanmugalingam, N., Regularity of quasi-minimizers on metric spaces, Manuscripta Math. 105 (2001), 401-423. 12

40. Korte, R., Geometric implications of the Poincaré inequality, Results Math. 50 (2007), 93-107. 10

41. Korte, R., Marola, N. and Shanmugalingam, N., Quasiconformality, homeomorphisms between metric measure spaces preserving quasiminimizers, and uniform density property, Ark. Math. 50 (2012), 111-134. 3

42. Koskela, P. and Macmanus, P., Quasiconformal mappings and Sobolev spaces, Studia Math. 131 (1998), 1-17. 4

43. LAAkso, T., Ahlfors $Q$-regular spaces with arbitrary $Q>1$ admitting weak Poincaré inequality, Geom. Funct. Anal. 10 (2000), 111-123; Erratum: Geom. Funct. Anal. 12 (2002), 650. 10

44. Li, X. and Shanmugalingam, N., Preservation of bounded geometry under sphericalization and flattening, Indiana Univ. Math. J. 64 (2015), 1303-1341. $2,6,7,10,21$

45. Llorente, J. G., Manfredi, J. J. and Wu, J.-M., p-harmonic measure is not additive on null sets, Ann. Sc. Norm. Super. Pisa Cl. Sci. 4 (2005), 357-373. 3, 20, 21, 22

46. NÄKKI, R., Boundary behavior of quasiconformal mappings in $n$-space, Ann. Acad. Sci. Fenn. Ser. A I Math. 484 (1970), 1-50. 17

47. Shanmugalingam, N., Newtonian spaces: An extension of Sobolev spaces to metric measure spaces, Rev. Mat. Iberoam. 16 (2000), 243-279. 8

48. Shanmugalingam, N., Harmonic functions on metric spaces, Illinois J. Math. 45 (2001), 1021-1050. 4

49. VÄIs̈̈LÄ, J., Lectures on n-dimensional Quasiconformal Mappings, Lecture Notes in Math. 229, Springer, Berlin-Heidelberg, 1971. 17

50. Wolff, T. H., Gap series constructions for the $p$-Laplacian, J. Anal. Math. 102 (2007), 371-394. 3, 21 